UCRL-ID-120838

\title{
Factors Affecting the Erosion of Jets Penetrating High Explosive
}

\section{Leonard C. Haselman \\ Kris A. Winer}

May 1, 1995

This is an informal report intended primarily for internal or limited external distribution. The opinions and conclusions stated are those of the authorand may or may not be those of the Laboratory.

Work performed under the auspices of the U.S. Department of Energy by the Lawrence Livermore National Laboratory under Contract W-7405-Eng-48. 
This document was prepared as an acccount of work sponsored by an agency of the United States Government. Neither the United States Government nor the University of California nor any of their employees, makes any warranty, express or implied, or assumes any legal liability or responsibility for the accuracy, completeness, or usefulness of any information, apparatus, product, or process disclosed, or represents that its use would not infringe privately own rights. Reference herein to any specific commercial products, process, or service by trade name, trademark, manufacturer, or otherwise, does not necessarily constitute or imply its endorsement, recommendation, or favoring by the United States Government or the University of California. The views and opinions of authors expressed herein do not necessarily state or reflect those of the United States Government or the University of California, and shall not be used for advertising or product endorsement purposes.

This report has been reproduced directly from the best available copy.

Available to DOE and DOE contractors from the Office of Scientific and Technical Information P.O. Box 62, Oak Ridge, TN 37831

Prices available from (615) 576-8401, FTS 626-8401

Available to the public from the National Technical Information Service

U.S. Department of Commerce 5285 Port Royal Rd. Springfield, VA 22161 


\section{DISCLAIMER}

Portions of this document may be illegible in electronic image products. Images are produced from the best available original document. 


\title{
Factors Affecting the Erosion of Jets Penetrating High Explosive
}

\author{
Leonard C. Haselman and Kris A. Winer
}

Introduction

It has been observed in various experiments with shaped charge jets penetrating high explosives that the erosion of the jet can be considerably greater than that expected from analytical theory or from two dimensional hydrodynamic computer simulations. In a previous study, ${ }^{1}$ we found that the initial penetration of the jet agreed with theory, and that the erosion of the jet happened subsequent to the initial penetration. This additional erosion can be the dominant factor in the total length of jet that is eroded. We also found that in one experiment the jet did not show any excess erosion and that the penetration could be predicted from theory. We also found a rough correlation of the amount of excess erosion with the diameter of the jet, with larger jet diameters giving less erosion.

A problem with previous experiments was that a wide variety of shaped charges, target shapes, and target thicknesses were used. This made it difficult to isolate the effect of a particular parameter. For the current study we chose to isolate the effects of scale and target thickness. For this purpose we used well characterized jets and carefully chosen targets. We also did computer calculations to help elucidate the underlying mechanisms of the excess erosion.

\section{Experimental Design and Results}

For our experiments we used two shaped charges, the TOW-2a and the Viper. Both have copper jets with similar tip velocities $(9.5 \mathrm{~mm} / \mu \mathrm{s})$ and mass distributions. The primary difference between the two is the size. The TOW-2a has a high explosive diameter of $146 \mathrm{~mm}$ and the Viper's is $65 \mathrm{~mm}$, for a scale factor of 2.25 . In a previous experiment with the TOW-2a shaped charge the jet penetrated a $60 \mathrm{~mm}$ long, $60 \mathrm{~mm}$ diameter cylinder of Comp-B at a standoff of 3 charge diameters (CD's) with no excess erosion. This was the only experiment that did not suffer excess erosion, and it served as the baseline for the current set of experiments. For the new experiments the diameter of the Comp-B cylindrical target was increased to $203 \mathrm{~mm}$, which was judged to be large enough to exclude any effect of the charge diameter on the results. The target thicknesses varied from 25 to $125 \mathrm{~mm}$ thick. All experiments were done at $3 \mathrm{CD}$ 's standoff, where the jets are still solid and the complications due to particulation of the jet are avoided.

Figure 1 shows the results of the baseline experiment, the TOW-2a into a $60 \mathrm{~mm}$ thick target. The tip of the jet is solid and is at the position expected from theoretical 
analysis. There is some very low density material in front of the solid tip which we have been unable to explain.

To investigate the effect of target thickness the TOW-2a charge was fired at a 125 $\mathrm{mm}$ thick target, roughly twice the thickness of the baseline experiment. Figure 2 shows the flash $X$-ray results of this experiment. There were considerable problems in protecting the $X$-ray cassette from shock damage when using the $125 \mathrm{~mm}$ target, which accounts for the pressure marks and poor quality of this X-ray. The TOW-2a suffered considerable excess erosion in penetrating the $125 \mathrm{~mm}$ target. This is characterized by lack of a distinct tip and the wispy appearance of the jet. The jet is shorter than would be expected from theory. The contrast between this experiment and the baseline is dramatic and illustrates the importance of the target thickness in determining the excess erosion of the jet.

To investigate the effect of scale we tested the Viper shaped charge against a 25 $\mathrm{mm}$ target. This target is a scale factor 2.4 smaller than the equivalent target used in the baseline TOW-2a experiment. This scale factors compares with the 2.25 scale factor of the TOW-2a to the VIPER. Thus the Viper experiment was an approximate scale of the TOW-2a experiments. The results of these experiments are shown in Figs. $3 \mathrm{a}$ and $3 \mathrm{~b}$. The radiograph of Fig. 3a was taken in the vertical plane of the shaped charge, while Fig. $3 \mathrm{~b}$ was in the horizontal plane. The results are similar to the TOW-2a baseline results. There is no excess erosion, although the jet has been deflected. These results indicate that there is little effect of scale on the HE erosion of jets. This contradicts our previous conclusions. If we include the HE target thickness in the analysis of our previous experiments the results are consistent with the conclusion that the dominant parameter controlling the erosion of the jet is the HE target thickness and not the scale of the shaped charge.

To confirm these results we did an additional experiment with the TOW-2a shaped charge. In this experiment we used a $50 \mathrm{~mm}$ thick target to provide a more precise comparison with the Viper experiment. This also provided a check on the sensitivity to minor variations in the target, since this experiment was similar to the baseline TOW-2a experiment. The results of this experiment are shown in Figs. 4a and $4 \mathrm{~b}$. The results of this experiment are similar to the results of the baseline experiment and like the Viper experiment, shows no excess erosion of the tip. It is interesting to note that the jet does not show any excess erosion even though the tip is not straight, as can be seen in the first exposure in Fig. 3a. This shows the dominance of the target thickness as the controlling parameter.

\section{Theoretical Considerations}

The fact that the erosion of the jet scales suggests that it is hydrodynamic in origin. In order to better understand this process we have done a series of hydrodynamic computer simulations. These are an idealized version of our experiments, which were designed to look at the differences in targets and not to be 
exact replications of the experiments. For these calculations we used a constant velocity rod rather than a stretching jet such as the Viper or TOW-2a. This was done to ensure that the penetration "hole" size did not depend upon the particular portion of the jet used and ensured that differences in the calculations would reflect the target differences. The velocity of the rod was $9.5 \mathrm{~mm} / \mu \mathrm{s}$, which is the tip velocity of the TOW-2a and the Viper. The diameter of the jet was $10 \mathrm{~mm}$. The TOW-2a diameter is $\sim 5 \mathrm{~mm}$ near the tip at $3 \mathrm{CD}$ 's elongation. Thus the calculations are about a factor of 2 scaling over the TOW-2a and 4 over the Viper. The diameter of the jet is used to determine the scale factor, since the target is sufficiently far from the jet source that the size of the source is irrelevant to the penetration processes.

Figures $5 \mathrm{a}$ and $5 \mathrm{~b}$ show the penetration of this rod into a semi-infinite medium at times of 20 and $32 \mu \mathrm{s}$. In these calculations the initial position of the rod tip was at $11 \mathrm{~cm}$ and the edge of the target was at $12 \mathrm{~cm}$. At $20 \mu \mathrm{s}$ the rod has penetrated $13 \mathrm{rod}$ diameters (RD's) into the high explosive. At this point in the penetration the explosive reaction products reattach to the jet at $\sim 5 \mathrm{RD}$ 's, with a region of high pressure on the rod of about $40 \mathrm{Kbars}$. The distance from the tip to the reattachment point is relatively constant throughout the calculation as seen in Fig. $4 \mathrm{~b}$. The main difference is that the pressure on the back part of the rod becomes higher and more extensive as the rod moves more deeply into the target, as is readily apparent in Fig. $4 \mathrm{~b}$. There is also considerable structure in this shock wave, which makes it difficult to assign a "characteristic" value to the pressure on the back part of the rod.

Figures $6 \mathrm{a}$ and $6 \mathrm{~b}$ show the same times for an identical calculation where the target is now $100 \mathrm{~mm}$ ( $10 \mathrm{RD}$ 's) in width. This case is roughly equivalent to the $25 \mathrm{~mm}$ target in the Viper and the $50 \mathrm{~mm}$ target in the TOW-2a experiments. In Fig. 5a the pressure distribution behind the rod tip is similar to the case of the semi-infinite calculation, since the rarefaction region from the face of the target at $22 \mathrm{~cm}$ is just beginning to affect this part of the rod. Fig. $5 \mathrm{~b}$ shows the rod at $32 \mu \mathrm{s}$. In this case the pressure acting on the back of the jet has decreased considerably. The region of elevated pressures at $\sim 15 \mathrm{~cm}$ is a stagnation point which is roughly equidistant from the front and back surfaces of the exploding detonation products.

If there is any asymmetry in this pressure on the jet, as could be caused by lack of straightness in the jet or an asymmetry in the target, then there will be a force acting perpendicular to the jet. This can cause a deflection of the jet tip as was seen in the Viper experiment. Once the jet is deflected it will have to repenetrate the HE products, leading to enhanced erosion. The amount of deflection will determine the amount of repenetration needed, and will depend upon the depth to which the jet has penetrated into the target.

The extent of this deflection and its dependence on the thickness of the target can be estimated as follows. First we note that there is an $\sim 5$ RD's length of jet behind the tip which sees no significant pressure from the penetration, followed by a length of jet which is acted upon by various pressures. We assume the jet is a constant velocity rod with velocity $V$, density $\rho$, and diameter $D$. If $P$ is the pressure of the HE products and 
$\Delta \mathrm{P}$ an asymmetry in this pressure, then the force acting on an effective cross section of the rod with an area Ddz, where dz is the length of an element of the jet, is $\triangle P D d z$. The acceleration of this element perpendicular to the jet is

$$
a=\frac{\Delta P D d z}{\rho \pi \frac{D^{2}}{4} d z} \approx \frac{\Delta P}{\rho D}
$$

If $\Delta P$ is an average which acts over a time $\delta t$, the velocity of deflection of the jet $\left(V_{d}\right)$ is

$$
\mathrm{V}_{\mathrm{d}}=\mathrm{a} \delta \mathrm{t}=\frac{\Delta \mathrm{P} \delta \mathrm{t}}{\rho \mathrm{DD}}
$$

If we define a significant deflection as being one jet diameter and that this deflection occurs in time $\delta \mathrm{t}$, then

$$
V_{d}=\frac{D}{\delta t}=\frac{\Delta P \delta t}{\rho D}
$$

from which we calculate the pressure asymmetry necessary to produce this deflection.

$$
\Delta P=\frac{\rho D^{2}}{\delta t^{2}}
$$

The time $\delta$ t during which the jet is deflected is dependent on both the depth of the target and the velocity of the jet. An element of the jet moves relative to the tip with a velocity

$$
v-v(1+\gamma)=v\left(\frac{\gamma}{1+\gamma}\right)
$$

where $\gamma$ is $\sqrt{\rho_{t} / \rho}$ and $\rho_{t}$ is the target density. Since the pressure field is attached to the eroding tip this is the appropriate velocity for $\delta$ t. The distance is just the target thickness minus the length behind the jet tip which is not subject to the pressure. Therefore $\delta t$ is

$$
\frac{\Delta \mathrm{Z}-5 \mathrm{D}}{\mathrm{V}\left(\frac{\gamma}{1+\gamma}\right)} \text { and } \Delta \mathrm{P}=\frac{\rho \mathrm{D}^{2} \mathrm{~V}^{2}\left(\frac{\gamma}{1+\gamma}\right)^{2}}{(\Delta \mathrm{Z}-5 \mathrm{D})^{2}}
$$


where $\Delta Z$ is the HE target thickness. In order to estimate the sensitivity to deflection we calculate the relative pressure

$$
\frac{\Delta \mathrm{P}}{\mathrm{P}}=\frac{\rho \mathrm{V}^{2}}{\mathrm{P}}\left(\frac{\gamma}{1+\gamma}\right)^{2}\left(\frac{\mathrm{D}}{\Delta \mathrm{Z}-\mathrm{5D}}\right)^{2} .
$$

This represents the relative pressure asymmetry necessary to produce a deflection of 1 rod diameter for a penetration of a target of depth $\Delta \mathrm{Z}$. The determination of the pressure $P$ is somewhat arbitrary. It can be obtained from computer simulations; however, the pressure is not constant and has considerable structure. The derivation here is designed primarily to show the functional dependence on the target thickness and various jet parameters. $P$ is generally chosen as a rough average over the length of the jet which is subject to perturbation. In general it increases with increasing $\Delta Z$.

For the TOW-2a with the $50 \mathrm{~mm}$ target and the Viper with the $25 \mathrm{~mm}$ target, where there is no excess erosion, $\Delta \mathrm{P}$ is $\sim 70 \mathrm{~Kb}$ and $\mathrm{P} \sim 30 \mathrm{~Kb}$. This gives a $\Delta \mathrm{P} / \mathrm{P}$ of $\sim 2$ which means that the asymmetry would need to be twice the actual pressure and therefore the deflection will be smaller than 1 rod diameter. For the TOW-2a against the $125 \mathrm{~mm}$ target, where there is significant erosion, $\Delta P$ is $\sim 6 \mathrm{~Kb}$ and $P \sim 60 \mathrm{~Kb}$. This gives a $\triangle P / P$ of .1 which means that a $10 \%$ asymmetry in the actual pressure is sufficient to deflect the jet 1 rod diameter. This analysis can also be applied to our previous data, and is successful in qualitatively predicting the excess erosion of the jet.

\section{Conclusions}

We have conducted a series of experiments which show that the phenomena of excess erosion of jets penetrating $\mathrm{HE}$ are scale independent. We have demonstrated a strong dependence of the erosion on the thickness of the target material and have derived the functional dependence for the deflection of a jet. These experiments and the related theory make it possible to estimate the difficulty that can be expected in penetrating an $\mathrm{HE}$ target.

\section{References}

1. L. Haselman, R. Jones, F. Kovar, K. Winer, "Erosion of Shaped-Charge Jets by High Explosives," UCRL-JC-113964 (1993). 


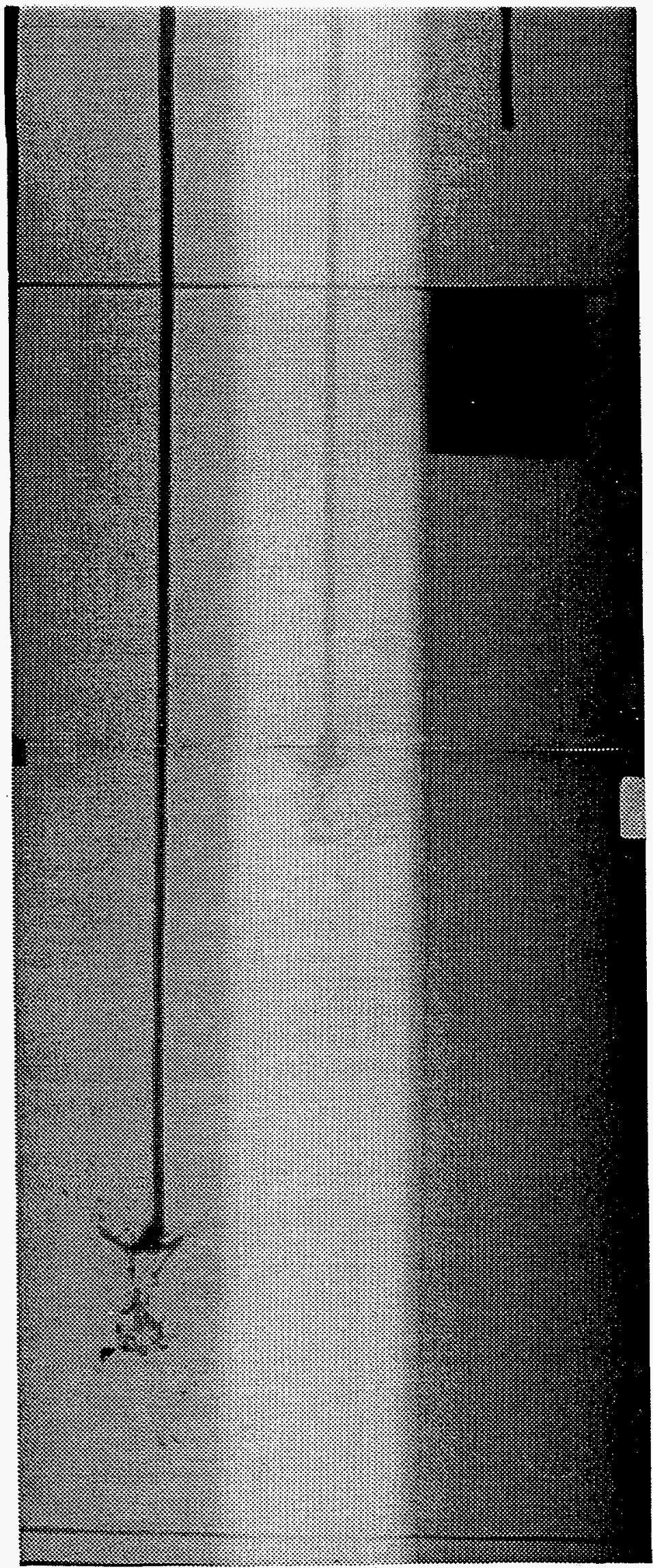

Fig. 1 Radiograph of TOW-2a shaped charge jet penetrating $60 \mathrm{~mm}$ Comp-B target.

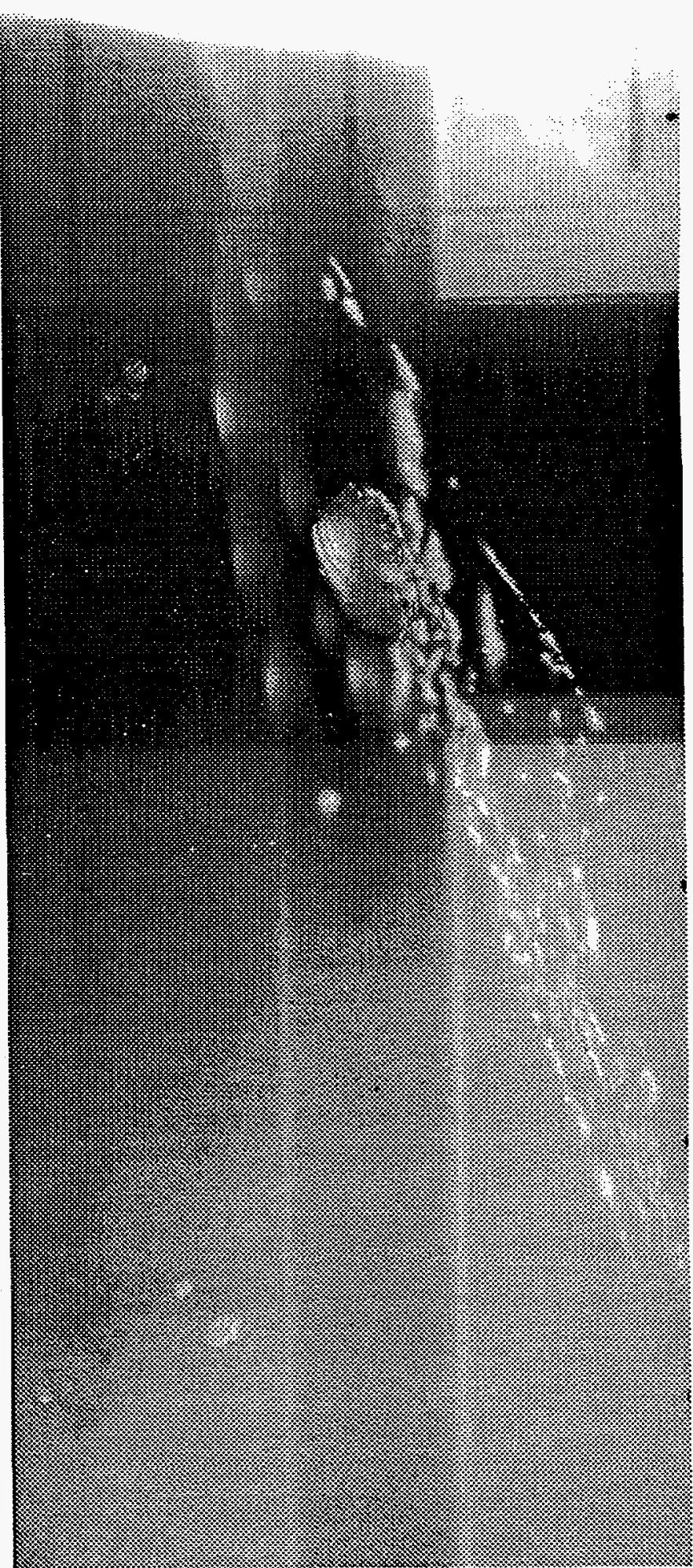

Fig. 2 Radiograph of TOW-2a shaped charge jet penetrating $125 \mathrm{~mm}$ Comp-B target. 

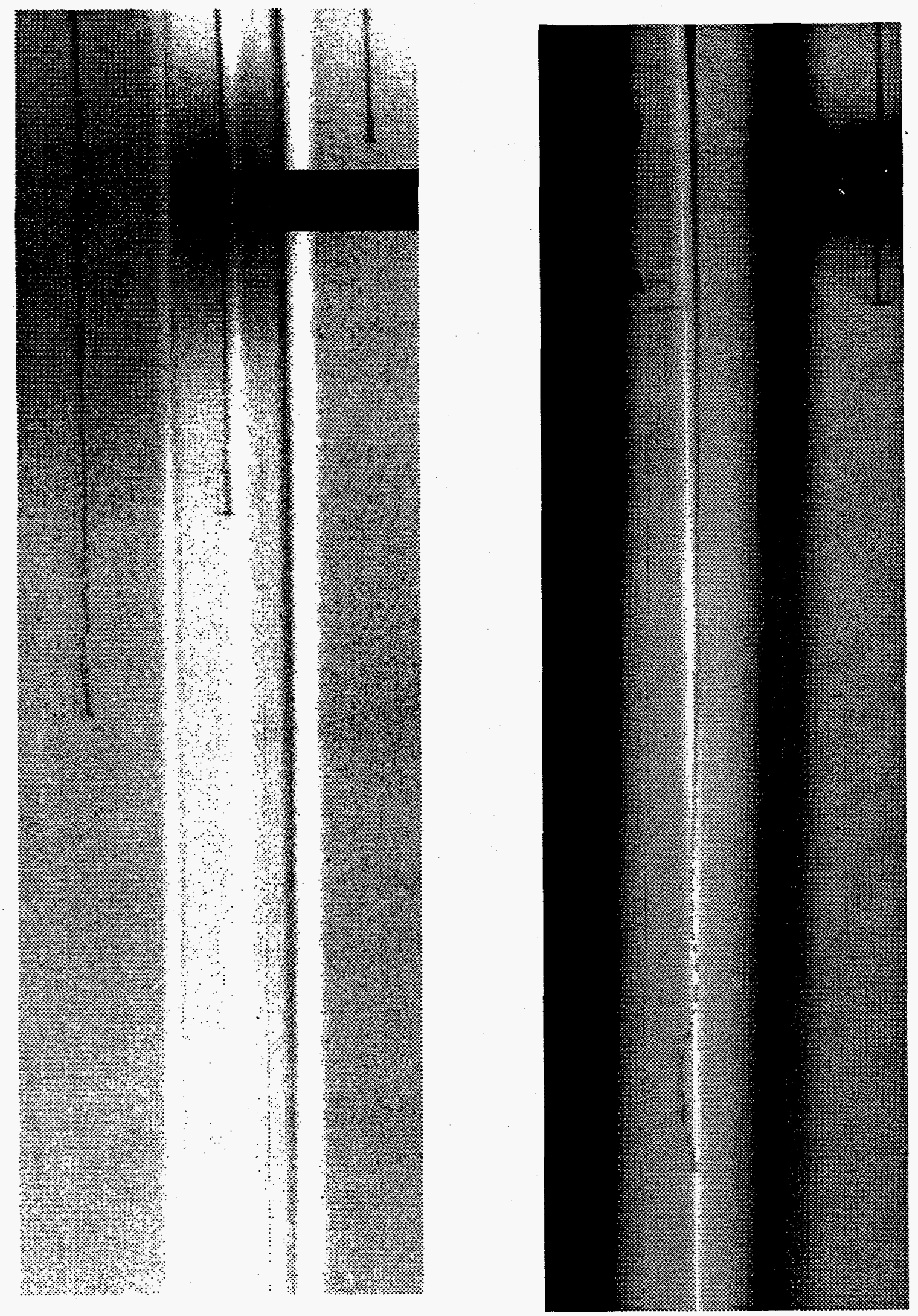

Fig. 3 Radiographs in vertical (a) and horizontal (b) planes of Viper shaped charge penetrating $25 \mathrm{~mm}$ Comp-B target. 

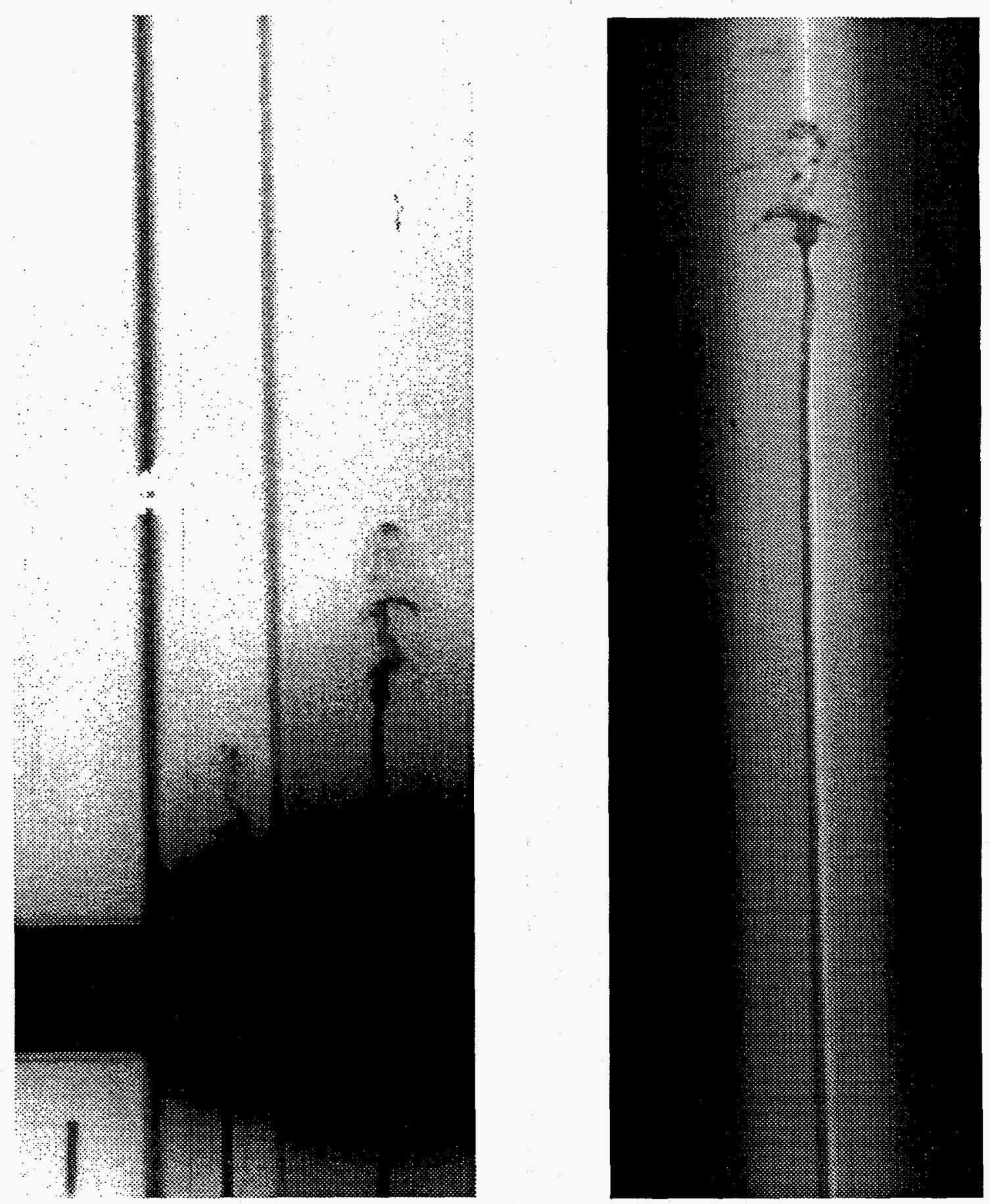

Fig. 4 Radiographs in vertical (a) and horizontal (b) planes of TOW-2a shaped charge jet penetrating $50 \mathrm{~mm}$ Comp-B target. 

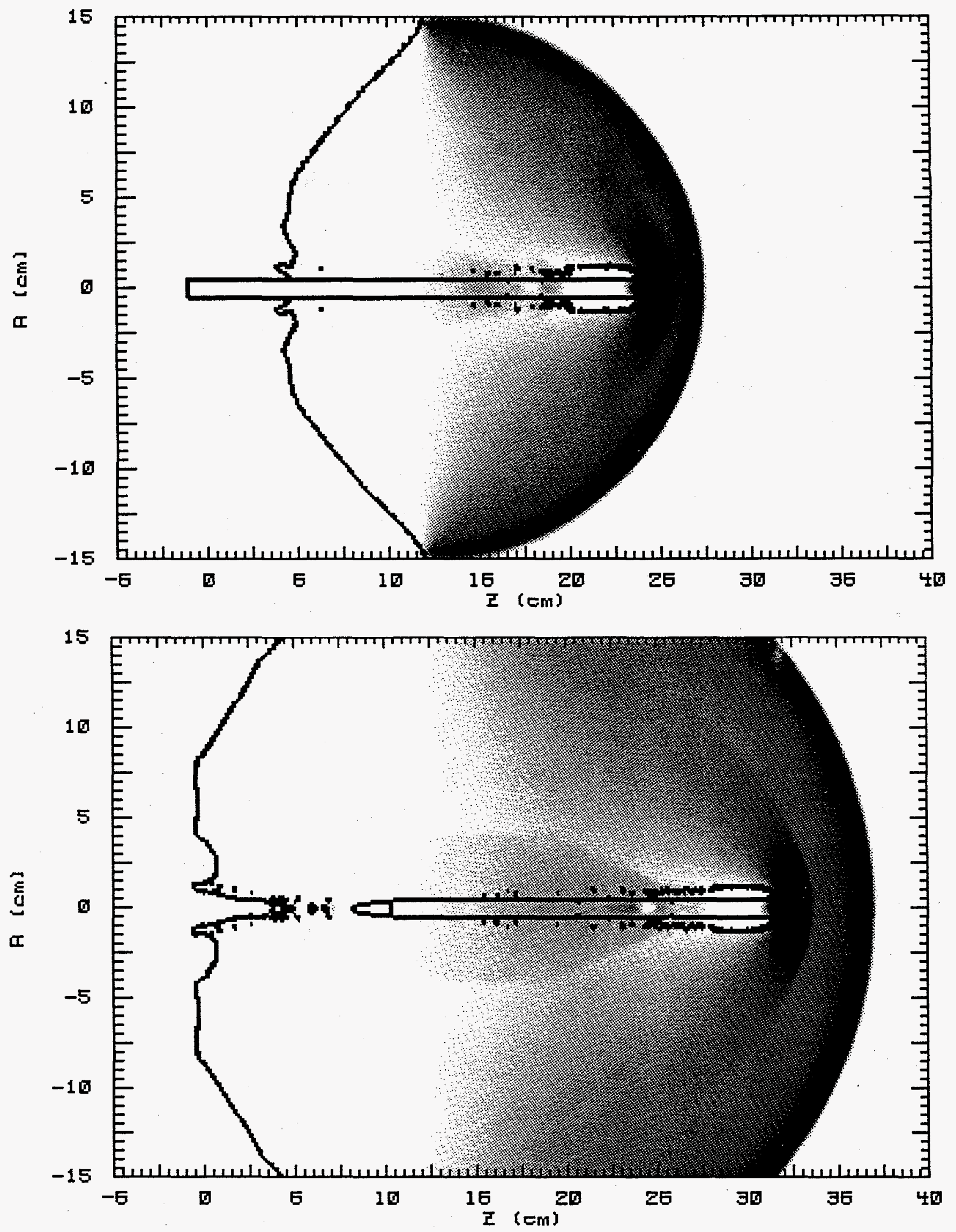

Fig. 5 Calculated pressure for penetration of a $1 \mathrm{~cm}$ diameter copper rod into semiinfinite Comp B at times of $20 \mu \mathrm{s}(\mathrm{a})$ and $32 \mu \mathrm{s}(\mathrm{b})$. Peak pressure is $200 \mathrm{Kbar}$. 

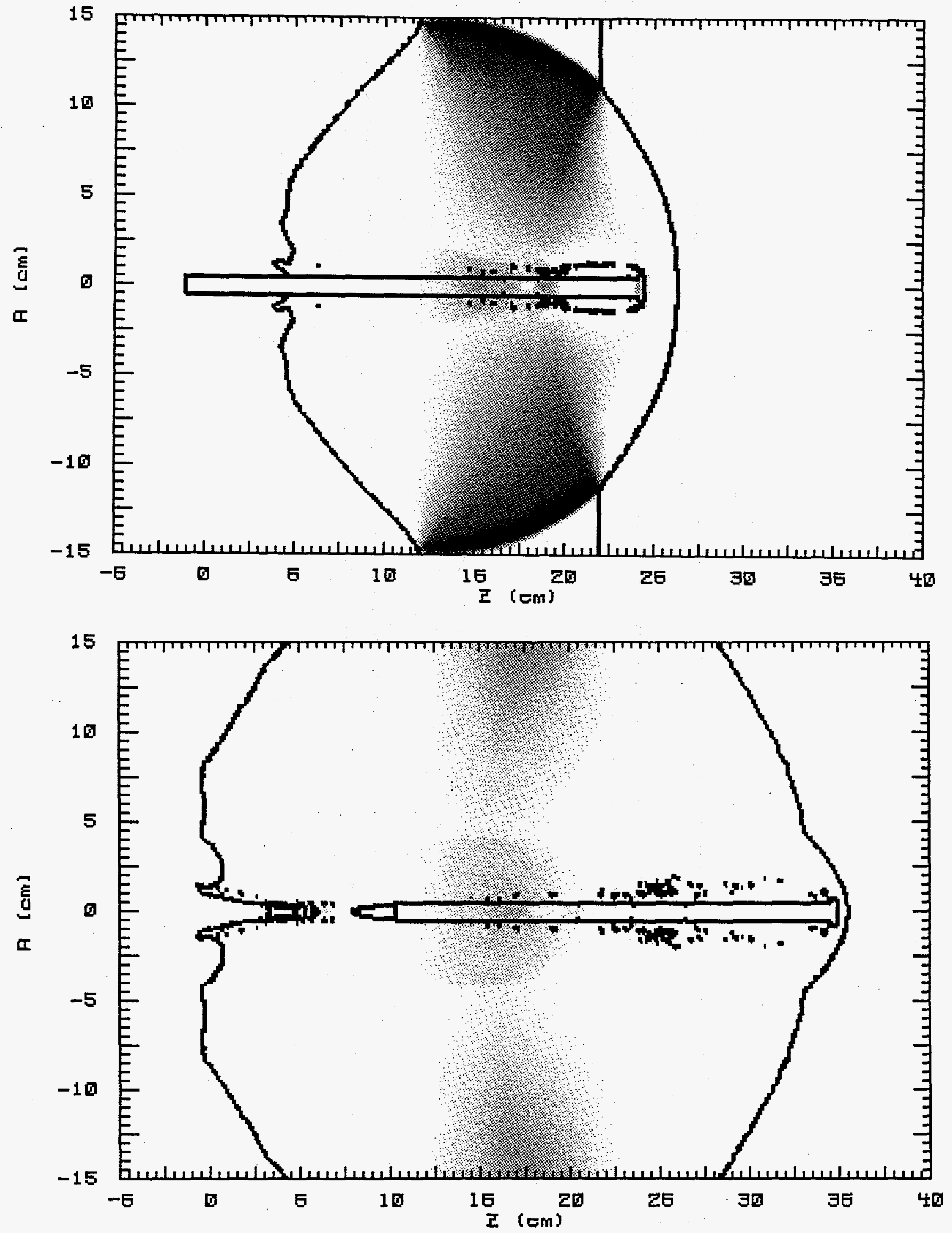

Fig. 6 Calculated pressure for penetration of a $1 \mathrm{~cm}$ diameter copper rod into a $10 \mathrm{~cm}$ thick Comp-B target at times of $20 \mu \mathrm{s}$ (a) and $32 \mu \mathrm{s}$ (b). Peak pressure is $200 \mathrm{Kbar}$. 